TTK-11-14

\title{
Self Regulation of Infrared Correlations for Massless Scalar Fields during Inflation
}

\author{
Björn Garbrecht and Gerasimos Rigopoulos \\ Institut für Theoretische Teilchenphysik und Kosmologie, \\ RWTH Aachen University, \\ D-52056 Aachen, Germany
}

\begin{abstract}
Self-energies of a minimally coupled scalar field with quartic and trilinear interactions are calculated in a de Sitter background, using a position space propagator. For quartic interactions, we recover earlier results for the seagull diagram, namely that it contributes an effective mass for the scalar field at leading order in the infrared enhancement in a steady-state de Sitter background. We further show that the sunset diagram also contributes to this effective mass and argue that these two contributions are sufficient in order to determine a self-consistent dynamical mass. In addition, trilinear interactions also induce a dynamical mass for the scalar field which we calculate. Since an interacting scalar field in de Sitter acquires a dynamical mass through these loop corrections, the infrared divergences of the two-point correlator are naturally self-regulated.
\end{abstract}

\section{Introduction}

It is well known that in de Sitter space, there is no propagator for a massless minimally coupled scalar field, which is of Hadamard form and that exhibits all ten space-time symmetries [1 13$]$ at the same time. The origin of this problem can be understood when constructing the propagator as an integral of free field modes. The integration over momentum space then leads to an infrared (IR) divergence. For this reason, one often refers to this issue as the IR problem of de Sitter space. ( $C f$. Ref. [4], where this and other matters are reviewed.) Under certain circumstances, this may lead to problems for models of the inflationary stage of cosmic expansion, where the space-time is approximately de Sitter. A plausible and pragmatic solution for the propagator during inflation relies on the assumption that inflation begins a finite number of e-folds $N_{\mathrm{e}}$ before it terminates. Then one may use a propagator that breaks de Sitter symmetry through an IR-enhanced term proportional to the logarithm of the scale factor or, equivalently, to the number of e-folds since the onset of inflation. This growing IR-term can be identified with the accumulation of superhorizon modes. 
The circumstances when the solution with the growing, de Sitter breaking propagator are no longer satisfactory are when $N_{\mathrm{e}}$ grows so large that the IR enhanced term in the propagator leads to a breakdown of perturbation theory in loop diagrams. The IR problem is therefore generic for models of inflation that assume a large number of e-foldings or even an infinite number, such as eternal inflation.

It is very plausible however that the IR problem is self-regulatory: A growing IR term for a scalar field $\phi(x)$ leads to a growing, dynamical mass $m_{\mathrm{dyn}}$ from the self-energy, which in turn limits the IR-enhanced term. To our knowledge, the viability of this idea has been demonstrated for a $\phi^{4}$ theory using three different approaches: stochastic inflation [5], Schwinger-Dyson equations [6] and the functional renormalisation group [7]. All these works agree on the result for the self-regulating, dynamical mass $m_{\mathrm{dyn}}$ that arises from the seagull diagram. A related approach has been developed for Euclidean de Sitter space, which is compact and therefore the IR divergence can be isolated in the discrete zero mode [8]. In the present work, we demonstrate that such an IR self-regularization does take place for scalar fields with trilinear and quartic interactions, and that for quartic interactions both the seagull and the sunset diagrams contribute to $m_{\mathrm{dyn}}$ and are sufficient to determine it.

The technical device we use to obtain our results is based on the Schwinger-Dyson equations in the Closed Time Path (CTP) formalism in a de Sitter background, which we introduce in Section 2. While these concepts are well known, we feel that their reiteration is necessary here in order to introduce the notations and conventions that are used in what follows. The first application, discussed in Section 3, is the seagull diagram of $\phi^{4}$ theory for which we reproduce the existing result [5 7], namely that the diagram generates a dynamical mass $m_{\mathrm{dyn}}$ whose value we determine through a simple self-consistency relation arising from the Schwinger-Dyson equations. Technically, the present approach differs from the one of Ref. [6] in that we use an expansion of the scalar propagator in powers of $m_{\mathrm{dyn}}^{2} / H^{2}$ that starts at order minus one, and that has been developed in Ref. [9]. Using this propagator, we avoid appealing to stochastic arguments and the Fokker-Planck equation for the calculation of the IR-enhancement effects as it is done in Ref. [6]. We note here, that the seagull correction is particularly simple, because it involves just one vertex, such that its effects are manifestly local and there are no memory integrals.

This simplification does not apply for trilinear interactions, discussed in Section 4 , and for which the self energy is non-local. However, we observe that the leading (negative order) contribution in $m_{\mathrm{dyn}}^{2} / H^{2}$ to the convolution integral between self energy and propagator appearing in the Schwinger-Dyson equations, is proportional to the retarded Green function of the covariant d'Alembertian operator. Acting with the d'Alembertian on the Schwinger-Dyson equations from the left yields local equations. This requires however the use of the ansatz of a free field with constant mass $m_{\text {dyn }}$ for the scalar field $\phi(x)$. The effectively local form is therefore only valid in time-independent situations, when the IR contribution is not evolving. Otherwise, the convolution integrals, which are sometimes referred to as memory integrals, cannot be simplified by this method. The main result of Section 4 is a self-consistency relation determining $m_{\mathrm{dyn}}$ for $\phi^{3}$ theory. 
We note that in order to determine the effective dynamical mass of spin- $\frac{1}{2}$ fermions in de Sitter space, a similar method as presented in Section 4 has been applied before in Ref. [10].

In Section 5, we return to a $\phi^{4}$ interaction and consider the 2-loop sunset diagram. Since two of the internal lines in the retarded self energy can be IR enhanced, the methods discussed for the trilinear interactions in the previous section can be applied here straightforwardly. Because of the simultaneous IR enhancement of two propagators, it turns out that the suppression due to the additional vertex is compensated when compared to the seagull diagram of section 3. Therefore, both corrections need to be included in the self-consistency relation that determines $m_{\text {dyn }}$ for $\phi^{4}$ theory and this is one of the main results of Section 5. Besides, we argue that diagrams at higher loop order only lead to subdominant contributions to $m_{\text {dyn }}$ at leading order in the IR enhancement. Section 6 is left for additional comments and conclusions.

Before closing the Introduction, we should note that the complete expressions for the self-energies in $\phi^{4}$ theory are given in Refs. [11-13]. In the present work, we do not give such a complete account of all terms to second order in the vertices of the selfenergy, but we only extract the leading contributions in $m_{\text {dyn }}^{2} / H^{2}$, which are of negative order and therefore IR enhanced. Taking account of these corrections, we show that there are self-consistent, de Sitter invariant equations for linear perturbations and for the propagators, that determine $m_{\text {dyn }}^{2}$. In this sense, Refs. [11 13] and the present work are complementary.

\section{Scalar Propagator in de Sitter Space and the CTP Formalism}

The models we consider are defined through the action

$$
S=\int d^{4} x \mathcal{L}
$$

where the Lagrangian is given by

$$
\mathcal{L}=\sqrt{-g}\left[g^{\mu \nu}\left(\partial_{\mu} \phi\right)\left(\partial_{\nu} \phi\right)-\frac{1}{2} m^{2} \phi^{2}\right]+\mathcal{L}_{\text {int }}+\delta \mathcal{L} .
$$

The first term is the Lagrangian for a free, massive minimally coupled scalar field $\phi(x)$. Throughout the present work, we assume that $\phi$ is light, by which we imply the relation $m \ll H$, where $H$ is the Hubble expansion rate. For loop corrections, this will lead to IR-enhanced terms $\sim H^{2} / m^{2}$ or $\sim H^{2} / m_{\text {dyn }}^{2}$, where $m_{\text {dyn }}$ is a dynamically generated mass that we require to satisfy $m_{\mathrm{dyn}} \ll H$ as well. Interaction terms are encompassed within $\mathcal{L}_{\text {int }}$. These give rise to ultraviolet (UV) divergences, which are renormalised by local counterterms that are contained in $\delta \mathcal{L}$. In the present work, we are interested in the effects of IR enhancements $\sim H^{2} / m^{2}$ or $\sim H^{2} / m_{\text {dyn }}^{2}$. Radiative corrections proportional 
to these enhancement factors turn out to be UV finite at leading order. We therefore omit a discussion of the UV divergences in the present context, leave the form of $\delta \mathcal{L}$ unspecified, and refer to Refs. [9 14] for detailed account of the concepts and technicalities of renormalisation in de Sitter background.

We use conformal coordinates $x^{\mu}$ with the metric tensor

$$
g_{\mu \nu}=a^{2} \eta_{\mu \nu}=a^{2} \operatorname{diag}(1,-1,-1,-1)
$$

The conformal time is given by $\eta=x^{0}$, and for de Sitter space, the scale factor is $a=-\frac{1}{H \eta}$ and $\eta \in[-\infty ; 0]$. By defining $\Delta x^{2}\left(x ; x^{\prime}\right)=x_{\mu} \eta^{\mu \nu} x_{\nu}^{\prime}$, the de Sitter invariant length function is

$$
y\left(x ; x^{\prime}\right)=a(\eta) a\left(\eta^{\prime}\right) H^{2} \Delta x^{2}=-4 \sin ^{2}\left(\frac{1}{2} H \ell\left(x ; x^{\prime}\right)\right),
$$

where we have indicated its relation with the geodesic distance $\ell\left(x ; x^{\prime}\right)$.

The free-field equation of motion obtained from the Lagrangian (2) is

$$
\begin{aligned}
& a^{4}\left(-\nabla_{x}^{2}-m^{2}\right) \phi(x) \\
= & -a^{2} \frac{d^{2}}{d \eta^{2}} \phi(x)-2 a\left(\frac{d a}{d \eta}\right) \frac{d}{d \eta} \phi(x)+a^{2} \vec{\nabla}^{2} \phi(x)-a^{4} m^{2} \phi(x)=0,
\end{aligned}
$$

and the equation for the free scalar propagator $\mathrm{i} \Delta^{(0)}\left(x ; x^{\prime}\right)$ is

$$
a^{4}\left(-\nabla_{x}^{2}-m^{2}\right) \mathrm{i} \Delta^{(0) f g}\left(x ; x^{\prime}\right)=f g \delta^{f g} \mathrm{i} \delta^{4}\left(x-x^{\prime}\right),
$$

where $\nabla_{x \mu}$ is the covariant derivative with respect to $x$. We define $z=1+\frac{y}{4}$ and $\{f, g\}=\{+,-\}$ are the CTP indices. Because of the de Sitter invariance, it is possible to express $a^{4} \nabla_{x}^{2}$ as an operator of $z$ and derivatives with respect to $z$ only (or, alternatively, $y)$. One finds that

$$
a^{4} H^{2}\left[z(1-z) \frac{d^{2}}{d z^{2}}+4\left(\frac{1}{2}-z\right) \frac{d}{d z}-\frac{m^{2}}{H^{2}}\right] \mathrm{i} \Delta^{(0) f g}\left(x ; x^{\prime}\right)=f g \delta^{f g} \mathrm{i} \delta^{4}\left(x-x^{\prime}\right) .
$$

The exact solution of this equation can be given in terms of a hypergeometric function. For the present purpose, we expand in $y$ and $m^{2} / H^{2}$ and find [9]

$$
\mathrm{i} \Delta^{(0) f g}\left(x, x^{\prime}\right)=\frac{H^{2}}{4 \pi^{2}}\left\{-\frac{1}{y^{f g}}-\frac{1}{2} \log \left(-y^{f g}\right)+\frac{3 H^{2}}{2 m^{2}}-1+\log 2+O\left(\frac{m^{2}}{H^{2}}\right)\right\},
$$

where $y^{f g}=a(\eta) a\left(\eta^{\prime}\right) H^{2} \Delta x^{f g^{2}}$. The term $\sim H^{2} / m^{2}$ within the curly brackets is the IR-enhancement factor. For comparison, the corresponding expression for a massless field is [1, 9]

$$
\mathrm{i} \Delta_{\mathrm{m}=0}^{f g}\left(x, x^{\prime}\right)=\frac{H^{2}}{4 \pi^{2}}\left\{-\frac{1}{y^{f g}}-\frac{1}{2} \log \left(-y^{f g}\right)+\frac{1}{2} \log \left(a(\eta) a\left(\eta^{\prime}\right)\right)-\frac{1}{4}+\log 2\right\} .
$$


The desired behaviour at the coincident point $x=x^{\prime}$ arises from the definitions

$$
\begin{aligned}
& \Delta x^{++2}\left(x ; x^{\prime}\right)=\left(\left|\eta-\eta^{\prime}\right|-\mathrm{i} \varepsilon\right)^{2}-\left|\mathbf{x}-\mathbf{x}^{\prime}\right|^{2}, \\
& \Delta x^{+-2}\left(x ; x^{\prime}\right)=\left(\eta-\eta^{\prime}+\mathrm{i} \varepsilon\right)^{2}-\left|\mathbf{x}-\mathbf{x}^{\prime}\right|^{2}, \\
& \Delta x^{-+^{2}}\left(x ; x^{\prime}\right)=\left(\eta-\eta^{\prime}-\mathrm{i} \varepsilon\right)^{2}-\left|\mathbf{x}-\mathbf{x}^{\prime}\right|^{2}, \\
& \Delta x^{--2}\left(x ; x^{\prime}\right)=\left(\left|\eta-\eta^{\prime}\right|+\mathrm{i} \varepsilon\right)^{2}-\left|\mathbf{x}-\mathbf{x}^{\prime}\right|^{2} .
\end{aligned}
$$

For a two-point function $G\left(x ; x^{\prime}\right)$ [in the present work, this can either be a free propagator $\Delta^{(0)}\left(x ; x^{\prime}\right)$, a full propagator $\Delta\left(x ; x^{\prime}\right)$ or the self-energy $\left.\Pi\left(x ; x^{\prime}\right)\right]$, we identify the Wightman-type functions

$$
G^{<}\left(x ; x^{\prime}\right)=G^{+-}\left(x ; x^{\prime}\right), \quad G^{>}\left(x ; x^{\prime}\right)=G^{-+}\left(x ; x^{\prime}\right),
$$

and the time-ordered and anti-time ordered functions

$$
G^{T}\left(x ; x^{\prime}\right)=G^{++}\left(x ; x^{\prime}\right), \quad G^{\bar{T}}\left(x ; x^{\prime}\right)=G^{--}\left(x ; x^{\prime}\right) .
$$

Moreover, we note that these can be combined to obtain the advanced and the retarded two-point functions as

$$
\begin{aligned}
& G^{A}=G^{T}-G^{>}=G^{<}-G^{\bar{T}}, \\
& G^{R}=G^{T}-G^{<}=G^{>}-G^{\bar{T}} .
\end{aligned}
$$

Applying these relations to the retarded and advanced propagators $\Delta^{(0) R, A}$, it is interesting to note that these do not exhibit the IR-enhancement and will depend on the mass only at the order $\mathrm{m}^{2} / \mathrm{H}^{2}$, which is beyond the scope of the approximations made within the present work.

The proper self-energy can be defined as

$$
\Pi^{a b}\left(x ; x^{\prime}\right)=\mathrm{i} \frac{\delta \Gamma_{2}[\Delta]}{\delta \Delta^{b a}\left(x^{\prime} ; x\right)},
$$

where $\Gamma_{2}$ is -i times the sum of all two particle-irreducible (2PI) vacuum diagrams with full propagators $\Delta$ as internal lines. The Schwinger-Dyson equations are

$$
a^{4}\left(-\nabla_{x}^{2}-m^{2}\right) \mathrm{i} \Delta^{a b}\left(x ; x^{\prime}\right)+\mathrm{i} c \int d^{4} w \mathrm{i}^{a c}(x ; w) \mathrm{i} \Delta^{c b}\left(w ; x^{\prime}\right)=\delta^{a b} \mathrm{i} \delta^{4}\left(x-x^{\prime}\right) .
$$

Note that these are generically non-linear, because $\Pi$ is a functional of $\Delta$. These equations are formally exact and describe the full real-time evolution of the quantum system, but due to their non-linearity approximations are needed in practice. Within the present work, we seek for the main corrections in terms of the IR enhancement factor $H^{2} / \mathrm{m}^{2}$ or $H^{2} / m_{\text {dyn }}^{2}$. Useful insight into the dynamics may also be gained form an effective equation of motion for the one-point function

$$
a^{4}\left(-\nabla_{x}^{2}-m^{2}\right) \phi(x)-\int d^{4} x^{\prime} \Pi^{R}\left(x ; x^{\prime}\right) \phi\left(x^{\prime}\right)=0,
$$

that can be used to obtain the response of the system to a small classical perturbation in the field $\phi(x)$. 


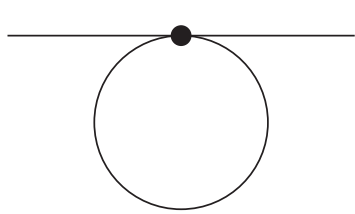

(A)

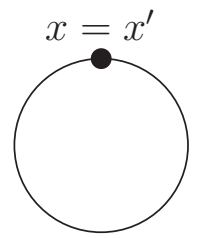

(B)

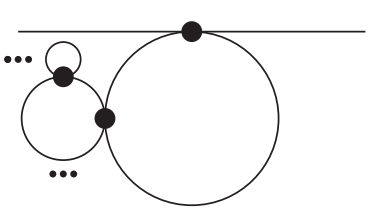

(C)

Figure 1: (A): Seagull diagram, (B): seagull contribution to the self-energy, (C): daisies/super-daisies

\section{Quartic Coupling: The Seagull Diagram}

In this Section, we consider the quartic self-interaction

$$
\mathcal{L}_{\text {int }}=-\sqrt{-g} \frac{\lambda}{4 !} \phi^{4} .
$$

The seagull contribution to the self energy is [cf. Figure1(B), where the propagator is is understood to be dressed]

$$
\Pi^{f g}\left(x, x^{\prime}\right)=\frac{\lambda}{2} \sqrt{-g(\eta)} \delta^{f g} \delta^{4}\left(x-x^{\prime}\right) \mathrm{i} \Delta^{f g}\left(x ; x^{\prime}\right) .
$$

This self energy is diagonal on the CTP, and therefore

$$
\begin{aligned}
& \Pi^{A}=\Pi^{T}-\Pi^{>}=\Pi^{<}-\Pi^{\bar{T}} \equiv \Pi^{T}, \\
& \Pi^{R}=\Pi^{T}-\Pi^{<}=\Pi^{>}-\Pi^{\bar{T}} \equiv \Pi^{T} .
\end{aligned}
$$

The UV divergences of $\mathrm{i} \Delta\left(x ; x^{\prime}\right)$ at the coincident point is cancelled by appropriate counterterms in $\delta \mathcal{L}$.

Let us now make the ansatz that the dressed propagator is $\Delta^{f g}\left(x ; x^{\prime}\right)$ takes the form of the propagator for a free field with a mass $m_{\text {dyn }}$ to be determined. This means that i $\Delta^{f g}\left(x ; x^{\prime}\right)$ satisfies

$$
a^{4}\left(-\nabla_{x}^{2}-m_{\mathrm{dyn}}^{2}\right) \mathrm{i} \Delta^{f g}\left(x ; x^{\prime}\right)=f g \delta^{f g} \mathrm{i} \delta^{4}\left(x ; x^{\prime}\right),
$$

implying in turn that $\Delta\left(x ; x^{\prime}\right)$ takes the form of Eq. (7) with $m^{2}$ replaced by $m_{\mathrm{dyn}}^{2}$. This ansatz therefore accounts for the IR enhancement to its leading orders $H^{2} / m_{\text {dyn }}^{2}$ and $\left(H^{2} / m_{\mathrm{dyn}}^{2}\right)^{0}$. Effectively, we assume that the growing term $\log \left(a(\eta) a\left(\eta^{\prime}\right)\right)$ in (8) which leads to an IR divergence and a breakdown of perturbation theory when used in loop diagrams, can be resummed into a mass term. Within the present approximation, this means that we can use

$$
\Pi^{T}=\Pi^{R}=\sqrt{-g} \lambda \frac{3 H^{4}}{16 \pi^{2} m_{\mathrm{dyn}}^{2}} \delta^{4}\left(x-x^{\prime}\right),
$$


as the appropriate approximation in order to extract the leading effects of the IR enhancements. Since this self energy is manifestly local and moreover diagonal on the CTP [cf. Eq. (20)], the Schwinger-Dyson equations take a very simple form, namely

$$
a^{4}\left(-\nabla_{x}^{2}-m^{2}\right) \mathrm{i} \Delta^{a b}\left(x ; x^{\prime}\right)-\sqrt{-g} \lambda \frac{3 H^{4}}{16 \pi^{2} m_{\text {dyn }}^{2}} \mathrm{i} \Delta^{a b}\left(x ; x^{\prime}\right)=\delta^{a b} \mathrm{i} \delta^{4}\left(x-x^{\prime}\right) .
$$

Taking this point of view and using (22), we obtain for $m_{\mathrm{dyn}}^{2}$

$$
m_{\mathrm{dyn}}^{2}=m^{2}+\lambda \frac{3 H^{4}}{16 \pi^{2} m_{\mathrm{dyn}}^{2}}
$$

such that

$$
m_{\mathrm{dyn}}^{2}=\frac{m^{2}}{2}+\sqrt{\frac{m^{4}}{4}+\lambda \frac{3 H^{4}}{16 \pi^{2}}} .
$$

For $m \rightarrow 0$, this reduces to

$$
m_{\mathrm{dyn}}^{2}=\frac{\sqrt{3 \lambda}}{4 \pi} H^{2}
$$

in agreement with Refs. [5 77]. Note that the requirement $m_{\mathrm{dyn}} \ll H$ is met provided $\lambda \ll 1$.

Turning our attention to the one-point function, we see that its effective equation of motion is consistent with the Schwinger-Dyson equation within our present approximations. Substituting Eq. (23) into the effective equation of motion (18) for $\phi$, we obtain

$$
a^{4}\left(-\nabla_{x}^{2}-m^{2}\right) \phi(x)-\sqrt{-g} \lambda \frac{3 H^{2}}{16 \pi^{2} m_{\mathrm{dyn}}^{2}} \phi(x)=0 .
$$

This equation can be solved by using a plane wave ansatz

$$
\phi(x)=-\frac{\nabla_{x}^{2}}{m_{\mathrm{dyn}}^{2}} \phi(x)+\phi(x) \times O\left(\frac{m_{\mathrm{dyn}}^{2}}{H^{2}}\right)
$$

from which we can directly deduce again Eqs. (25, 27).

In closing this Section, we note that the solution of the Schwinger-Dyson equation including the seagull correction in terms of the dressed propagator effectively resums the daisy and superdaisy corrections in terms of the bare propagators, as it is indicated in Figure1(C). Moreover, the Schwinger-Dyson equations resum all one-particle irreducible (1PI) contributions to the self-energies. Besides, one should expect that certain additional higher-loop order diagrams contribute to a complete self-consistent determination of $m_{\mathrm{dyn}}$ [15]. When promoting $\phi(x)$ to a globally $O(N)$-symmetric field, these higher order diagrams are generically suppressed by powers of $1 / N$ [5 7, 16, 17]. In Section [5, we demonstrate however, that even without resorting to $1 / N$ expansion, there is only one remaining diagram that contributes to $m_{\text {dyn }}$ at leading order in the IR-enhancement, which is the sunset diagram. 


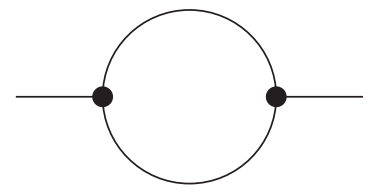

(A)

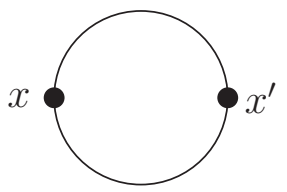

(B)

Figure 2: (A): Triscalar correction to the propagator, (B): triscalar self energy.

\section{Triscalar Coupling}

In order to distinguish better between the cause and the effect of the dynamical mass term, we first introduce an additional light minimally coupled scalar field $\chi$ of mass $m_{\chi}$, that couples to $\phi$ through the interaction

$$
\mathcal{L}_{\text {int }}=-\sqrt{-g} \frac{h_{\chi}}{2} \phi \chi^{2} .
$$

Let us first look at the one-point function. The self energy of $\phi$ is [cf. Figure 2 (B)]

$$
\mathrm{i} \Pi^{f g}\left(x ; x^{\prime}\right)=\frac{1}{2} h_{\chi}^{2} \sqrt{-g(\eta)} \sqrt{-g\left(\eta^{\prime}\right)} \mathrm{i} \Delta_{\chi}^{f g}\left(x ; x^{\prime}\right) \mathrm{i} \Delta_{\chi}^{f g}\left(x ; x^{\prime}\right) .
$$

The leading contribution in orders of $m_{\chi}^{2} / H^{2}$ to the retarded self-energy is

$$
\Pi^{R}\left(x ; x^{\prime}\right)=\frac{1}{2} h_{\chi}^{2} \sqrt{-g(\eta)} \sqrt{-g\left(\eta^{\prime}\right)} \frac{3 H^{4}}{4 \pi^{2} m_{\chi}^{2}} \bar{\Delta}_{\chi}^{R}\left(x ; x^{\prime}\right)+O\left[\left(\frac{m_{\chi}^{2}}{H^{2}}\right)^{0}\right] .
$$

Note that the retarded propagator $\bar{\Delta}_{\chi}^{R}$ is not identical to $\Delta_{\chi}^{R}$, but it deviates as

$$
\bar{\Delta}_{\chi}^{R}=\Delta_{\chi}^{R}+H^{2} \times O\left(\frac{m_{\chi}^{2}}{H^{2}}\right) .
$$

To the level of our approximations, i.e. the expansion in $m_{\chi}^{2} / H^{2}$, we may use

$$
\mathrm{i} \bar{\Delta}_{\chi}^{R}=\frac{H^{2}}{4 \pi^{2}}\left\{-\frac{1}{y_{++}}+\frac{1}{y_{+-}}-\frac{1}{2} \log \left(y_{++}\right)+\frac{1}{2} \log \left(y_{+-}\right)\right\} .
$$

The bar therefore indicates that it is accurate up to order $\left(m_{\chi}^{2} / H^{2}\right)^{0}$.

Substituting this into Eq. (18) and using the methods employed e.g. in Ref. [9] does not lead to a local effective equation, however. We obtain

$$
a^{4}\left(-\nabla_{x}^{2}-m^{2}\right) \phi(x)-\frac{1}{2} h_{\chi}^{2} \frac{3 H^{4}}{4 \pi^{2} m_{\chi}^{2}} a^{4} \int d^{4} x^{\prime} \sqrt{-g\left(x^{\prime}\right)}\left[\bar{\Delta}^{R}\left(x ; x^{\prime}\right)+O\left(m_{\chi}^{2}\right)\right] \phi\left(x^{\prime}\right)=0 .
$$


The covariant d'Alembertian derivative acting on $\bar{\Delta}_{\chi}^{R}$ is well defined,

$$
-a^{4} \nabla_{x}^{2} \bar{\Delta}_{\chi}^{R}\left(x ; x^{\prime}\right)=\delta^{4}\left(x-x^{\prime}\right),
$$

which implies that $\bar{\Delta}_{\chi}^{R}$ is the retarded Green function for $-a^{4} \nabla_{x}^{2}$. When substituting the plane wave ansatz (29), we can perform the integral in Eq. (35) by parts and obtain

$$
\begin{aligned}
a^{4}\left(-\nabla_{x}^{2}-m^{2}\right) \phi(x) & -\frac{1}{2} h_{\chi}^{2} \frac{3 H^{4}}{4 \pi^{2} m_{\chi}^{2}} \frac{1}{m_{\mathrm{dyn}}^{2}} \phi(x) \\
& +\frac{1}{2} h_{\chi}^{2} \frac{H^{4}}{4 \pi^{2} m_{\chi}^{2}} a^{4} \int d^{4} x^{\prime} \frac{1}{m_{\mathrm{dyn}}^{2}}\left[\nabla_{x}^{2} O\left(m_{\chi}^{2}\right)\right] \phi\left(x^{\prime}\right)=0 .
\end{aligned}
$$

The last term originates from the approximation (33) and it is subdominant compared to the second one by a factor of $m_{\chi}^{2} / H^{2}$, when assuming that $m_{\chi}^{2} / H^{2} \ll 1$ and $m_{\text {dyn }}^{2} \sim m_{\chi}^{2}$.

The plane wave solution (29) therefore must satisfy

$$
m_{\mathrm{dyn}}^{2}=m^{2}+\frac{1}{2} h_{\chi}^{2} \frac{3 H^{4}}{4 \pi^{2} m_{\chi}^{2}} \frac{1}{m_{\mathrm{dyn}}^{2}} .
$$

Replacing the interaction Lagrangian (30) by the cubic interaction

$$
\mathcal{L}_{\text {int }}=-\sqrt{-g} \frac{h}{3 !} \phi^{3}
$$

effectively leads to the replacements $m_{\chi}^{2} \equiv m_{\text {dyn }}^{2}$ and $h_{\chi} \rightarrow h$. We then obtain an equation for a self-consistent mass:

$$
m_{\mathrm{dyn}}^{6}-m^{2} m_{\mathrm{dyn}}^{4}-\frac{1}{2} h^{2} \frac{3 H^{4}}{4 \pi^{2}}=0 .
$$

When $m$ is negligibly small, this implies

$$
m_{\mathrm{dyn}}^{2}=\left(\frac{3}{8 \pi^{2}}\right)^{1 / 3} h^{2 / 3} H^{4 / 3} .
$$

In order to meet the condition $m_{\text {dyn }} \ll H$, we have to require here that $h \ll H$.

An alternative way to derive these results is to act on Eq. (35) with $a^{4} \nabla_{x}^{2} a^{-4}$ from the left. This approach is similar to the one pursued in Ref. [10] for Yukawa interactions. We obtain

$$
a^{4}\left(-\nabla_{x}^{2}-m^{2}\right) \nabla_{x}^{2} \phi(x)-a^{4} \frac{1}{2} h^{2} \frac{3 H^{4}}{4 \pi^{2} m_{\chi}^{2}} \phi(x)=0 .
$$

Substituting again the plane-wave ansatz (29) leads to Eq. (37).

The above results for $m_{\mathrm{dyn}}^{2}$ can also be obtained by considering the Schwinger-Dyson equations for the propagator. However, in contrast to the one-loop self energy from the 
quartic coupling, the self energy from the triscalar coupling is non-local and we therefore need to modify the procedure for solving the Schwinger-Dyson equations. It is useful here to split these into the Kadanoff-Baym equations and equations for the retarded and advanced propagators as

$$
\begin{aligned}
& a^{4}\left(-\nabla_{x}^{2}-m^{2}\right) \mathrm{i} \Delta^{<,>}\left(x ; x^{\prime}\right)=-\mathrm{i} \int d^{4} w \mathrm{i}^{R}(x ; w) \mathrm{i} \Delta^{<,>}\left(w, x^{\prime}\right) \\
& -\mathrm{i} \int d^{4} w \mathrm{i}^{<,>}(x ; w) \mathrm{i} \Delta^{A}\left(w, x^{\prime}\right), \\
& a^{4}\left(-\nabla_{x}^{2}-m^{2}\right) \mathrm{i} \Delta^{R, A}\left(x ; x^{\prime}\right)=\mathrm{i} \delta^{4}\left(x ; x^{\prime}\right)-\mathrm{i} \int d^{4} w \mathrm{i} \Pi^{R, A}(x ; w) \mathrm{i} \Delta^{R, A}\left(w, x^{\prime}\right) .
\end{aligned}
$$

Due to the $\delta$-function on the right-hand side, acting with the d'Alembertian on the equations for the retarded and advanced propagators does not lead to a useful simplification. We recall from Section 2 however that the retarded and advanced propagators do not contain information about the mass at the leading orders $\left(\mathrm{m}^{2} / \mathrm{H}^{2}\right)^{-1}$ and $\left(m^{2} / H^{2}\right)^{0}$, such that we do not need these equations in order to determine the selfconsistent mass $m_{\text {dyn }}$. The Kadanoff-Baym equation (43b) can be reduced to a local form when acting with the covariant d'Alembertian $a^{4} \nabla_{x}^{2} a^{-4}$ from the left. Note that $\nabla_{x}^{2} a^{-4} \Pi^{<,>}=h^{2} H^{6} \times O(1)$, such that this term is negligible compared to the leading self-energy correction $\nabla_{x}^{2} \int d^{4} w \Pi^{R}(x, w) \sim h^{2} H^{4} / m_{\mathrm{dyn}}^{2}$ when $m_{\mathrm{dyn}} \ll H$. In analogy with Eq. (42), we obtain

$$
a^{4}\left(-\nabla_{x}^{2}-m^{2}\right) \nabla_{x}^{2} \Delta^{<,>}\left(x ; x^{\prime}\right)-a^{4} \frac{1}{2} h^{2} \frac{3 H^{4}}{4 \pi^{2} m_{\text {dyn }}^{2}} \Delta^{<,>}\left(x ; x^{\prime}\right)=0 .
$$

Again, the plane wave ansatz $\left(-\nabla_{x}^{2}-m_{\mathrm{dyn}}^{2}\right) \Delta^{<,>}\left(x ; x^{\prime}\right)=0$ then leads to the selfconsistency relations (40, 41) for $m_{\mathrm{dyn}}$.

We emphasise that this local form holds only for the plane-wave ansatz with timeindependent $m_{\mathrm{dyn}}^{2}$. In general, we expect that during the time when the IR terms are accumulating as $\log \left(a(x) a\left(x^{\prime}\right)\right)$, the ansatz of a time-independent $m_{\mathrm{dyn}}$ should not be valid and the memory integrals cannot be simplified to an effectively local form.

In order to compare with the earlier works on the self-energies of photons [9] and of chiral fermions [10], which are conformally coupled particles, we conclude this Section by considering the situation when $\phi$ is conformally coupled to the expanding background, which can be achieved by adding the term $-\frac{1}{6} \sqrt{-g} R \phi^{2}$ to the Lagrangian (2), where $R$ is the Ricci scalar, which takes the value $R=12 H^{2}$ in de Sitter space. We again couple to a minimally coupled light $\left(m_{\chi} \ll H\right)$ field $\chi$ through the interaction (30).

The effective equation of motion then reads

$$
a^{4}\left(-\nabla_{x}^{2}-m^{2}-\frac{1}{6} R\right) \phi(x)-\int d^{4} x^{\prime} \Pi^{R}\left(x ; x^{\prime}\right) \phi(x)=0,
$$

with $\Pi$ as in Eqs. (31, 32). Acting with $a^{4} \nabla_{x}^{2} a^{-4}$ from the left, we obtain

$$
a^{4}\left(-\nabla_{x}^{2}-m^{2}-\frac{1}{6} R\right) \nabla_{x}^{2} \phi(x)-a^{4} \frac{1}{2} h_{\chi}^{2} \frac{3 H^{4}}{4 \pi^{2} m_{\chi}^{2}} \phi(x)=0 .
$$


The plane-wave ansatz for a conformally coupled, massive field

$$
\left[-\nabla_{x}^{2}-\frac{1}{6} R\right] \phi(x)=m_{\mathrm{dyn}}^{2} \phi(x)
$$

leads to

$$
\left(m_{\mathrm{dyn}}^{2}-m^{2}\right)\left(m_{\mathrm{dyn}}^{2}+\frac{1}{6} R\right)=\frac{1}{2} h_{\chi}^{2} \frac{3 H^{4}}{4 \pi^{2} m_{\chi}^{2}} .
$$

For $m^{2} \ll m_{d y n}^{2}$ and $m_{\text {dyn }}^{2} \ll H^{2}$, we find

$$
m_{\mathrm{dyn}}^{2}=h_{\chi}^{2} \frac{3 H^{2}}{16 \pi^{2} m_{\chi}^{2}} .
$$

Compared to the mass-square for the minimally coupled scalar field (38) with $m=0$, the right hand side differs by a factor of $m_{\text {dyn }}^{2} / H^{2}$. It is therefore interesting to compare this result with earlier ones obtained for conformally coupled fields interacting with a light, minimally coupled scalar. For a photon $\gamma$ coupled to a scalar field with charge $e$ and mass $m_{\chi}$, the mass-square is

$$
m_{\gamma}^{2}=\frac{3 e^{2} H^{4}}{4 \pi^{2} m_{\chi}^{2}},
$$

while for a pair of Weyl fermions $\psi_{L, R}$ with a Yukawa coupling $f$ to a real scalar field of mass $m_{\chi}$

$$
m_{\psi}^{2}=\frac{3 f^{2} H^{4}}{8 \pi^{2} m_{\chi}^{2}}
$$

is found for the dynamical effective mass-square. Up to a replacement of a factor $H^{2} \rightarrow h_{\chi}^{2}$, that takes place for dimensional reasons, the result (49) for a conformally coupled scalar field fits well into one scheme with the earlier results for photons (50) and fermions (51), which are both conformally coupled as well, while Eq. (38) for the mass square of the minimally coupled scalar field exhibits an interesting difference.

\section{Quartic Coupling: The Sunset Diagram}

The methods for finding the effective self-consistent mass for the cubic interaction can also be applied to evaluate the leading contribution to the sunset diagram of $\lambda \phi^{4}$ in terms of an effective mass term. This is because the leading contribution to the retarded self-energy contains the IR-enhancement term to quadratic order and again, the retarded Green function of the covariant d'Alembertian as additional factor. Explicitly, the leading $\left(\sim 1 / m_{\text {dyn }}^{4}\right)$ contribution to the self-energy is [cf. Figure $\left.3(B)\right]$

$$
\mathrm{i} \Pi^{R}\left(x ; x^{\prime}\right)=\frac{\lambda^{2}}{2}\left(\frac{3 H^{2}}{8 \pi^{2} m_{\mathrm{dyn}}^{2}}\right)^{2} \mathrm{i} \bar{\Delta}^{R}\left(x ; x^{\prime}\right)
$$




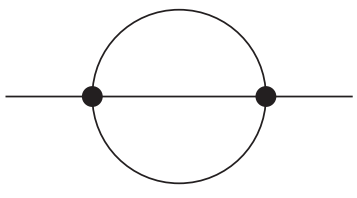

(A)

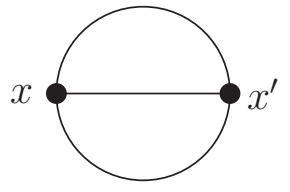

(B)

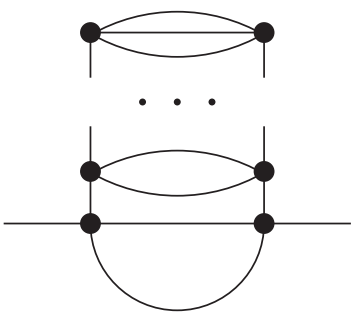

(C)

Figure 3: (A): Sunset diagram, (B): sunset contribution to the self-energy, (C): ladder diagram.

and therefore, using the plane wave ansatz (29)

$$
\int d^{4} x^{\prime} \Pi^{R}\left(x ; x^{\prime}\right) \phi\left(x^{\prime}\right)=\frac{\lambda^{2}}{2} a^{4}\left(\frac{3 H^{4}}{8 \pi^{2} m_{\mathrm{dyn}}^{2}}\right) \frac{1}{m_{\mathrm{dyn}}^{2}} \phi(x) .
$$

This contribution is to be combined with the one-loop result from Eq. (28), what leads to the self-consistency relation

$$
\frac{\lambda^{2}}{2}\left(\frac{3 H^{4}}{8 \pi^{2} m_{\mathrm{dyn}}^{2}}\right)^{2} \frac{1}{m_{\mathrm{dyn}}^{2}}+\lambda \frac{3 H^{4}}{16 \pi^{2} m_{\mathrm{dyn}}^{2}}=a^{4} m_{\mathrm{dyn}}^{2} .
$$

We find

$$
m_{\mathrm{dyn}}^{2}=\frac{\sqrt{3 \lambda} H^{2}}{2 \sqrt{2} \pi} .
$$

Note that both, the one-loop and the sunset diagram contribute at the same order in $\lambda$. This relation can also be obtained from Schwinger-Dyson equations, in complete analogy with Section 4, such that we do not repeat this discussion here.

We note that the iterative addition of two rungs to the sunset diagram leads to additional ladder diagrams as indicated in Figure 3(C). Each such insertion is suppressed by a factor of $\lambda^{2}$ from the new vertices, but it is also enhanced by a factor of $\lambda^{2}$ from the IR-enhanced contributions of the four new propagators, which are $\sim 1 / \sqrt{\lambda}$ each. However, these ladder diagrams are not part of the proper self-energy. This is most easily seen from the definition (16). The diagram contributing to $\Gamma_{2}$, which gives rise to the sunset contribution to $\Pi$, is $2 \mathrm{PI}$, whereas the vacuum ladder diagrams are two-particle reducible and therefore not part of $\Gamma_{2}$. We therefore conclude that the ladder diagrams are readily resummed within the self-consistency relation (55) (along with super ladders arising from ladder insertions into internal lines).

By this counting argument, according to which each vertex contributes a factor $\lambda$ and each propagator a factor $1 / \sqrt{\lambda}$, the diagrams in Figure 4 might be expected to contribute to the self-consistency relation that determines $m_{\mathrm{dyn}}$ at the leading (negative) 


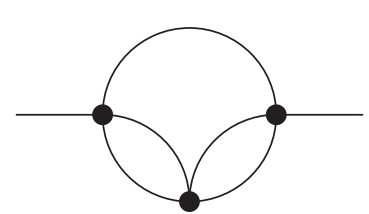

(A)

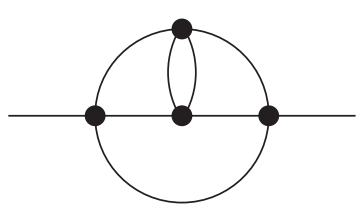

(B)

Figure 4: Diagram (A) contributes at NLO in the $1 / N$ expansion, diagram (B) at NNLO. However, the IR-enhanced contributions from these diagrams are suppressed in the expansion in $m_{\mathrm{dyn}}^{2} / H^{2}$.

order in $m_{\text {dyn }}^{2} / H^{2}$ as well, cf. e.g. Ref. [15]. When the value of the propagator is nonperturbatively large, one can consider a theory with an $O(N)$ symmetric field $\phi(x)$ and perform a $1 / N$ expansion [16, 17]. In this situation, the seagull graph of Figure 1 (A) is leading order $[\mathrm{LO}, O(1)]$. The sunset diagram and an infinite series of diagrams that starts with the one in Figure 4 (A) contributes at next to leading order [NLO, $O(1 / N)]$. The diagram of Figure 4 (B) is one of infinitely many diagrams that contribute at the next-to next-to leading order [NNLO, $\left.O\left(1 / N^{2}\right)\right]$. While it is possible to sum all NLO diagrams in such a theory, we notice that this is not necessary in the present case, because the diagrams in Figure 4 do not contribute to the retarded self-energy at leading (negative) order in $m_{\mathrm{dyn}}^{2} / H^{2}$ : At the leading order in $m_{\mathrm{dyn}}^{2} / H^{2}$, all except for one propagator are IR enhanced. This is because when all propagators are IR enhanced, the summation over CTP indices \pm (taking account of a factor of \pm 1 for these vertices and of the fact that the IR enhanced term is identical for all four CTP propagators) immediately leads to a vanishing result. By the same token, if the remaining nonIR-enhanced propagator is not directly connected to the right external vertex of the self-energy, the CTP summation again leads to a vanishing contribution to the retarded self-energy. Now, if the single non-IR-enhanced propagator is connected to an internal vertex and the right external vertex, the identity $\Delta^{++}+\Delta^{--}-\Delta^{+-}-\Delta^{-+}=0$ leads to a vanishing contribution to the retarded self-energy. Finally, we consider the case where the non-IR-enhanced propagator connects to both external vertices. If there are internal vertices, the CTP sum leads to a vanishing term again. Only in the case of the sunset diagram, where there are no internal vertices, a non-vanishing contribution remains.

\section{Discussion and Conclusions}

In this work, we have calculated retarded self-energies for scalar fields with quartic and trilinear interactions at leading order in the IR enhancement. One of the main results is Eq. (55), which can be interpreted as the effective self-consistent mass that a scalar field with quartic self-interaction acquires in a steady-state de Sitter space. Self consistent masses during inflation have been determined earlier in Refs. [5 7]. In the present paper, we have used the propagator (7), which has been proposed in Ref. [9] and which perhaps leads to simplifications when compared to earlier treatments. Moreover, 
we have determined the effective self-mass for self-energies, that are non-local, i.e. for the triscalar interaction and for the sunset diagram. For this purpose, we have made a plane-wave ansatz in a steady-state de Sitter background (22)29), which allows to reduce the effective equations of motion for the propagator and for the one-point function to a local form, cf. Eqs. (37/42]44]46). In turn, this implies that provided the system is not in a steady state [substantial deviations from de Sitter and/or deviations from the plane wave form (22]29) for the scalar field], there remain in principle still non-local terms in the form of memory integrals. In contrast, the self-energy from the seagull diagram (20) is always manifestly local. This perhaps explains differences between the cases of a quartic and a trilinear self-interaction, that have been observed in Ref. [7] when using functional renormalisation group methods.

The approximation strategies employed in the present work appear related to those of Ref. [8] for a scalar field on a four-dimensional sphere. Because the sphere is compact, there is a discrete zero mode. The leading self-consistent propagator is obtained when including the loop effects of the zero mode (including its own self-interactions), while neglecting the loop effects of the remaining modes. Here, we extract the leading contributions from the IR enhanced term $3 H^{4} / 8 \pi^{2} m^{2}$ (which may be mapped to the zero mode on the sphere) within the loop diagrams. While both approaches appear to be very similar, there is still a difference in the numerical coefficient of $m_{\mathrm{dyn}}$. It would be interesting to resolve the origin of this discrepancy.

We briefly estimate whether the self-regulatory mass is sufficient to bar large IR effects on $(\lambda / 4 !) \phi^{4}$ inflation. From Eq. (55), we immediately see that the dynamical mass is too small to change the phenomenology of this model of inflation, because $\lambda \sim 10^{-14}$ and consequently $m_{\mathrm{dyn}} \ll H$ by orders of magnitude. Using Eqs. (77) and (55), we can estimate the IR enhanced term [the third term on the right hand side of Eq. (77)] as

$$
\left\langle\phi^{2}\right\rangle_{\mathrm{IR}} \approx \sqrt{\frac{3}{2}} \frac{H^{2}}{2 \sqrt{\lambda}} .
$$

Since $H=\sqrt{8 \pi / 3} \sqrt{(\lambda / 4 !) \phi^{4} / m_{\mathrm{Pl}}^{2}}$ and $\langle\phi\rangle=O\left(m_{\mathrm{Pl}}\right)$ during inflation, the IR enhanced term (56) is $\sim \sqrt{\lambda} m_{\mathrm{Pl}}^{2}$, which is much smaller than $\langle\phi\rangle^{2}$. Finally, it is interesting to notice that if we suppose $\left\langle\phi^{2}\right\rangle_{\mathrm{IR}} \approx 0$ initially and that it subsequently grows as $\left[H^{2} /\left(8 \pi^{2}\right)\right] N_{\mathrm{e}}$ (as may be motivated from Refs. [1-3]) it takes $N_{\mathrm{e}} \approx 2 \sqrt{6} \pi / \sqrt{\lambda}$ e-folds before saturating to the value (56). We emphasize that these are rough estimates in particular due to the fact that the self-mass (55) only applies to a steady state, while here we have discussed properties of an evolving system. Sizeable effects from the IR enhancement may yet be expected in some multi-field models of inflation or small field models with a large number of e-folds.

In conclusion, the present work shows that there is a self-regulatory dynamical mass for $\phi^{4}$ theory in de Sitter which suppresses the IR enhanced terms. The only leading IR enhanced contributions to the retarded self-energy originate from the seagull and the sunset diagrams and can be calculated in a self-consistent way. It would be interesting to apply the techniques presented here to study some aspects of gravitational interactions in de Sitter space as well as cosmological perturbations in slow-roll inflation. 


\section{Acknowledgements}

The authors would like to thank Martin Beneke, Emanuela Dimastrogiovanni and David Seery for useful discussions. This work is supported by the Gottfried Wilhelm Leibniz programme of the Deutsche Forschungsgemeinschaft.

\section{References}

[1] B. Allen, "Vacuum States in de Sitter Space," Phys. Rev. D32 (1985) 3136.

[2] B. Allen, A. Folacci, "The Massless Minimally Coupled Scalar Field In De Sitter Space," Phys. Rev. D35 (1987) 3771.

[3] N. C. Tsamis, R. P. Woodard, "The Physical basis for infrared divergences in inflationary quantum gravity," Class. Quant. Grav. 11, 2969-2990 (1994).

[4] D. Seery, "Infrared effects in inflationary correlation functions," Class. Quant. Grav. 27 (2010) 124005. arXiv:1005.1649 [astro-ph.CO]].

[5] A. A. Starobinsky, J. Yokoyama, "Equilibrium state of a selfinteracting scalar field in the De Sitter background," Phys. Rev. D50 (1994) 6357-6368. [astro-ph/9407016].

[6] A. Riotto, M. S. Sloth, "On Resumming Inflationary Perturbations beyond Oneloop," JCAP 0804 (2008) 030. [arXiv:0801.1845 [hep-ph]].

[7] C. P. Burgess, L. Leblond, R. Holman, S. Shandera, "Super-Hubble de Sitter Fluctuations and the Dynamical RG," JCAP 1003 (2010) 033. arXiv:0912.1608 [hep-th]].

[8] A. Rajaraman, "On the proper treatment of massless fields in Euclidean de Sitter space," Phys. Rev. D82 (2010) 123522. [arXiv:1008.1271 [hep-th]].

[9] T. Prokopec, E. Puchwein, "Photon mass generation during inflation: de Sitter invariant case," JCAP 0404 (2004) 007. astro-ph/0312274].

[10] B. Garbrecht, T. Prokopec, "Fermion mass generation in de Sitter space," Phys. Rev. D73 (2006) 064036. gr-qc/0602011].

[11] V. K. Onemli, R. P. Woodard, "Superacceleration from massless, minimally coupled $\phi^{4}, "$ Class. Quant. Grav. 19 (2002) 4607. gr-qc/0204065.

[12] V. K. Onemli, R. P. Woodard, "Quantum effects can render $w<-1$ on cosmological scales," Phys. Rev. D70 (2004) 107301. gr-qc/0406098.

[13] T. Brunier, V. K. Onemli, R. P. Woodard, "Two loop scalar self-mass during inflation," Class. Quant. Grav. 22 (2005) 59-84. [gr-qc/0408080].

[14] B. Garbrecht, "Ultraviolet Regularisation in de Sitter Space," Phys. Rev. D74 (2006) 043507. hep-th/0604166]. 
[15] C. P. Burgess, R. Holman, L. Leblond, S. Shandera, "Breakdown of Semiclassical Methods in de Sitter Space," JCAP 1010 (2010) 017. [arXiv:1005.3551 [hep-th]].

[16] J. Berges, "Controlled nonperturbative dynamics of quantum fields out-ofequilibrium," Nucl. Phys. A699 (2002) 847-886. [hep-ph/0105311].

[17] J. Berges, "Introduction to nonequilibrium quantum field theory," AIP Conf. Proc. 739 (2005) 3-62. [hep-ph/0409233]. 